\title{
Rapid response systems in Korea
}

\author{
Bo Young Lee', Sang-Bum Hong² \\ 'Division of Allergy and Respiratory Diseases, Department of Internal Medicine, Soonchunhyang University Hospital, Seoul;'2Department of Pulmonary and \\ Critical Care Medicine, Asan Medical Center, University of Ulsan College of Medicine, Seoul, Korea
}

The inpatient treatment process is becoming more and more complicated with advanced treatments, aging of the patient population, and multiple comorbidities. During the process, patients often experience unexpected deterioration, about half of which might be preventable. Early identification of patient deterioration and the proper response are priorities in most healthcare facilities. A rapid response system (RRS) is a safety net to identify antecedents of these adverse events and to respond in a timely manner. The RRS has become an essential part of the medical system worldwide, supported by all major quality improvement organizations. An RRS consists of a trigger system and response team and needs constant assessment and process improvement. Although the effectiveness and cost-benefit of RRS remain controversial, according to previous studies, it may be beneficial by decreasing in-hospital cardiac arrest and mortality. Since the first implementation of RRS in Korea in 2008, it has been developed in over 15 medical centers and continues to expand. Recent accreditation standards and an RRS pilot program by the Korean government will promote the proliferation of RRSs in Korea.

Key Words: hospital medical emergency team; hospital rapid response team; patient safety

\section{Review Article}

Received: May 12, 2019

Revised: May 17, 2019

Accepted: May 20, 2019

\section{Corresponding author}

Sang-Bum Hong

Department of Pulmonary and Critical Care Medicine, Asan Medical Center, University of Ulsan College of Medicine, 88 Olympic-ro 43-gil, Songpa-gu, Seoul 05505, Korea Tel: +82-2-3010-3130

Fax: +82-2-3010-6968

E-mail: sbhong@amc.seoul.kr

\section{INTRODUCTION}

It is well known that approximately $10 \%$ of patients admitted to hospital experience unexpected serious adverse events [1]. A rapid response system (RRS) is a patient safety strategy that prevents cardiac arrests or deaths by providing immediate and timely interventions when patients unexpectedly deteriorate $[2,3]$. Delayed or inappropriate medical management in these patients may result in an increased risk of death or disability. An RRS aims to improve the safety of hospital-ward patients. Although the effectiveness of RRSs is controversial [4,5], several before-and-after studies have shown a reduction in cardiac arrest and hospital mortality [6-11]. Starting in the United States and Australia, this system has become an essential part of patient safety and has been adopted worldwide. Last year, the Korea Institute for Healthcare Accreditation distributed its third edition revised accreditation standards, and it recommended all acute care hospitals implement an RRS. In addition, in May 2019, the Korean Health Insurance Review and Assessment Service and the Ministry of Health and Welfare started a rapid response system pilot program. This article reviews the concept of the RRS; its requirements; and its past, present, and future in Korea.

Copyright () 2019 The Korean Society of Critical Care Medicine

This is an Open Access article distributed under the terms of Creative Attributions Non-Commercial License (http:// creativecommons.org/li-censes/by-nc/4.0/) which permits unrestricted noncommercial use, distribution, and reproduction in any medium, provided the original work is properly cited. 


\section{RAPID RESPONSE SYSTEM}

\section{Patient Safety Issues}

Admitted patients experience unexpected adverse events in about $10 \%$ of cases, $7.3 \%$ of which could be fatal. Although not all unexpected adverse events are preventable or predictable, $30 \%$ to $83 \%$ are so $[1,12,13]$. Approximately $80 \%$ of in-hospital cardiorespiratory arrest showed at least one abnormal sign such as blood pressure, heart rate, respiratory rate, body temperature, or change in consciousness within 8 hours before the event [14-16]. This suggests that there is an opportunity for intervention before patient condition worsens. However, these antecedents can go unrecognized in general wards. In most hospitals, continuous monitoring of vital signs is usually available only in the intensive care unit (ICU). The number of physician rounds for ward-patients is one to two times a day. The interval between measurements of vital signs is usually 8 hours or longer. Respiratory rate and mental state should be measured under direct observation, which is prone to error $[15,17]$. Even if there is a warning sign, it may not be recognized or properly alerted depending on personal experience, attitude, work environment, and position of the responding nurse or the doctor. As there is a long chain of command to activatefrom nurse to intern or resident, from resident to fellow, fellow to attending physician - an alert may be delayed in each step [2].

In the surgical ward, doctors are not readily available because they might be in the operating room. Even if the patient's warning signs are recognized and reported in a timely manner, it can be difficult to provide appropriate monitoring, intervention, or treatment because of the limited medical resources available in general wards. The Surviving Sepsis Campaign emphasizes early recognition and treatment. Accordingly, the current guideline combines 3- and 6-hour bundles into a "1-hour bundle" to emphasize immediate resuscitation and management [18]. Considering this, the current conventional medical system might be inadequate for proper early response.

\section{Components of RRS}

An RRS is composed of doctors and nurses who specialize in intensive care medicine to cope with patients at risk. An RRS consists of an afferent limb, efferent limb, patient safety, quality improvement with feedback from data analysis, and administrative components including education of staff [3], as shown in Figure 1. The afferent limb is a way to detect patient deterioration [19]. A nurse or a ward-physician can contact the responding team according to "calling criteria." Alterna-

\section{KEY MESSAGES}

- A rapid response system (RRS) is a patient safety system that prevents adverse events by providing immediate interventions when patients unexpectedly deteriorate.

- Several studies have shown that implementation of an RRS results in reduction of unexpected mortality and cardiac arrest.

- In Korea, more than 15 hospitals have implemented an RRS, and this is expected to increase in the near future.

tively, using scores such as the modified Early Warning Score (MEWS), patients at risk can be actively screened [20,21]. As under-recognition of monitored abnormal values leads to delay in activation, a surveillance system using a scoring system like MEWS (Table 1) [20] or the National Early Warning Score (NEWS) (Figure 2) [22] can be useful for detecting unexpected adverse events.

Once deterioration has been detected, the responding team works as an efferent limb. The forms may vary-for example, doctor and nurse can work as a team, or the nurse can respond first, and the doctor can cooperate when necessary. According to the composition and character of the responding team, it can be referred to as a rapid response team (RRT), medical emergency team (MET), critical care outreach (CCO) program, or critical care transit program. An RRT is often a nurse-led team. An MET is usually physician-led, offering several critical care interventions such as airway management, central vascular line insertion, and resuscitation. A CCO program

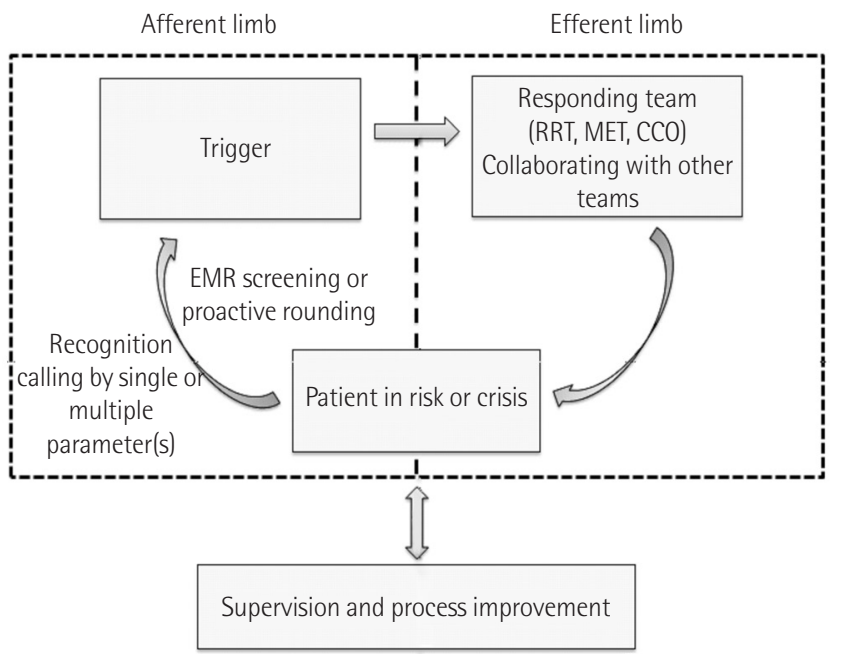

Figure 1. Structure of the rapid response system. RRT: rapid response team; MET: medical emergency team; CCO: critical care outreach; EMR: electronic medical recording. 
Table 1. The modified Early Warning Score (MEWS)

\begin{tabular}{|c|c|c|c|c|c|c|c|}
\hline & 3 & 2 & 1 & 0 & 1 & 2 & 3 \\
\hline Systolic blood pressure (mmHg) & $\leq 70$ & $71-80$ & $81-100$ & 101-199 & & $\geq 200$ & \\
\hline Heart rate (bpm) & & $\leq 40$ & $41-50$ & $51-100$ & $101-110$ & $111-129$ & $\geq 130$ \\
\hline Respiratory rate (bpm) & & $<9$ & & $9-14$ & $15-20$ & $21-29$ & $\geq 30$ \\
\hline Temperature $\left({ }^{\circ} \mathrm{C}\right)$ & & $<35.0$ & & $35-38.4$ & & $\geq 38.5$ & \\
\hline AVPU score & & & & Alert & Reacting to voice & Reacting to pain & Unresponsive \\
\hline
\end{tabular}

Reproduced from Subbe et al. OJM 2001;94:521-6, with permission of Oxford University Press [20].

AVPU: $A$, alert; $V$, responding to voice; $P$, responding to pain; $U$, unresponsive.

\begin{tabular}{|c|c|c|c|c|c|c|c|}
\hline \multirow{2}{*}{$\begin{array}{c}\text { Physiological } \\
\text { parameter }\end{array}$} & \multicolumn{7}{|c|}{ Score } \\
\hline & 3 & 2 & 1 & 0 & 1 & 2 & 3 \\
\hline $\begin{array}{l}\text { Respiration rate } \\
\text { (per minute) }\end{array}$ & $\leq 8$ & & $9-11$ & $12-20$ & & $21-24$ & $\geq 25$ \\
\hline $\mathrm{SpO}_{2}$ scale $1(\%)$ & $\leq 91$ & $92-93$ & $94-95$ & $\geq 96$ & & & \\
\hline $\mathrm{SpO}_{2}$ scale $2(\%)$ & $\leq 83$ & $84-85$ & $86-87$ & $\begin{array}{c}88-92 \\
\geq 93 \text { on air }\end{array}$ & $\begin{array}{c}\text { 93-94 on } \\
\text { oxygen }\end{array}$ & $\begin{array}{c}\text { 95-96 on } \\
\text { oxygen }\end{array}$ & $\begin{array}{l}\geq 97 \text { on } \\
\text { oxygen }\end{array}$ \\
\hline Air or oxygen? & & Oxygen & & Air & & & \\
\hline $\begin{array}{l}\text { Systolic blood } \\
\text { pressure }(\mathrm{mmHg})\end{array}$ & $\leq 90$ & $91-100$ & $101-110$ & $111-219$ & & & $\geq 220$ \\
\hline Pulse (per minute) & $\leq 40$ & & $41-50$ & $51-90$ & $91-110$ & $111-130$ & $\geq 131$ \\
\hline Consciousness & & & & Alert & & & CVPU \\
\hline Temperature $\left({ }^{\circ} \mathrm{C}\right)$ & $\leq 35.0$ & & $35.1-36.0$ & $36.1-38.0$ & $38.1-39.0$ & $\geq 39.1$ & \\
\hline
\end{tabular}

Figure 2. Data from Royal College of Physicians [22]. The National Early Warning Score (NEWS) scoring system. CVPU: C, new confusion; V, responds to voice; $P$, responds to pain; $U$, unresponsive.

adds active surveillance for patients at risk to RRT, managing high-risk patients in advance. The responding team should be equipped with real-time monitoring devices, vascular access devices, oxygen delivery devices, and intervention medications [3].

Continuous quality improvement including self-assessment and data analysis with feedback is another important part in RRS. This quality improvement limb is essential to prevent or prepare for future events. To determine event rates, resource requirements and outcomes, indicators such as number of calls, reason for call, unit where the call started, time of each call, number of transfers to ICU, and the number of calls converted to cardiopulmonary resuscitation (CPR) are collected. Monthly meeting for review of potentially avoidable cases would be an effective strategy for quality improvement.

\section{Implementation of RRS in Other Countries}

The Institute for Healthcare Improvement started the "100,000 Lives Campaign" in 2004 to engage U.S. hospitals to prevent 100,000 needless inpatient deaths. To reach this goal, the Institute for Healthcare Improvement suggested six clinical interventions including deployment of an RRT. About 3,100 hospitals (about 3/4 of all hospitals in the United States) were enrolled in the campaign and prevented an estimated 122,300 needless deaths [23]. Sixty percent of hospitals enrolled in the campaign implemented an RRS to detect and intervene in those in crises. As a result, cardiac arrest rate in the general ward was reduced by $50 \%$, transfer from ward to ICU decreased by $58 \%$, and overall mortality decreased by $37 \%$. The Institute for Healthcare Improvement followed with the 5 Million Lives Campaign, and related campaigns were launched in Canada, 
Australia, Sweden, Denmark, and the UK.

This system is widely supported by accreditors and quality improvement organizations such as the Joint Commission in the USA. The 2009 Joint Commission's National Patient Safety Goal recommended implementation of an RRS [24]. The 2015 American Heart Association Guidelines for Cardiopulmonary Resuscitation and Emergency Cardiovascular Care also recommended it to reduce the incidence of cardiac arrest, particularly in the general care ward [25]. As a result, an RRS is now implemented in more than 3,700 hospitals throughout the United States and has spread to other continents including Europe and Asia [2,4,26-28].

The preferred composition of the responding team differs among countries and institutions. In the UK, the RRS team may be nurse-led [29], and in the USA, nurses or respiratory therapists may lead [27]. In Australia, New Zealand, and Scandinavia, a physician-led RRS is favored [26,30].

\section{Effectiveness of RRS}

The Medical Early Response Intervention and Therapy (MERIT) trial [4] was a multicenter, cluster-randomized controlled trial of MET, which failed to demonstrate benefit regarding composite endpoint of death, unexpected cardiac arrest, and unplanned ICU admission. However, in the MERIT trial, the cardiac arrest response team was utilized in the control arm about $50 \%$ of the time, even before the actual event of cardiac arrest. This may have decreased the gap between the control and the intervention arm with RRS. In the past, the effects of RRS were controversial, but in recent years, there have been solid results supporting this system. Many observational be- fore-and-after studies showed that RRS resulted in decreased in-hospital mortality and cardiac arrest [6-11,30]. The study by Salvatierra et al. [11] was an observational cohort study including 471,062 adult patients from 2001 to 2009. It revealed that RRS improved in-hospital mortality by $24 \%$ compared to the pre-RRT time period. Another recent retrospective study found a significant decrease in unexpected and overall mortality [10]. Table 2 is a summary of studies reporting in-hospital mortality $[4,7,11,26,27,29-32]$. Although the studies mentioned above show considerable heterogeneity, a meta-analysis by Maharaj et al. [33] has consistently shown similar effectiveness of RRS. Implementation of RRS in the adult population was associated with a reduction of overall hospital mortality and cardiorespiratory arrest. In a tertiary hospital in Korea (Asan Medical Center), we found that the number of inhospital cardiac arrests decreased with increase of RRS calls (Figure 3). Based on these results, implementation of RRSs and annual RRT calls are increasing [34].

\section{Past and Present of RRS in Korea}

Despite the lack of accurate data related to patient safety events in the Korean medical system, one study found that about $7 \%$ of patients experienced at least one adverse event, and that $61 \%$ of events were preventable. In Korea, use of an RRS started in 2008, and the number of hospitals operating an RRS has since been increasing. Asan Medical Center first started an RRS in March 2008; this response team was called the medical alert team (MAT). At first, this system was applied to limited patients as a pilot project. It then was expanded to every patient in the hospital in May 2009. In addition to a calling sys-

Table 2. Summary of mortality in studies evaluating rapid response systems

\begin{tabular}{|c|c|c|c|c|c|}
\hline \multirow{2}{*}{ Study } & \multicolumn{2}{|c|}{ Sample size } & \multicolumn{2}{|c|}{ Mortality rate per 1,000 admissions (\%) } & \multirow{2}{*}{$\begin{array}{l}\text { OR of death } \\
(95 \% \mathrm{Cl})\end{array}$} \\
\hline & Control group & RRT group & Control group & RRT group & \\
\hline \multicolumn{6}{|c|}{ Observational and before-and-after study } \\
\hline Buist et al. [31] & 19,317 & 22,847 & 19.7 & 17.2 & $0.87(0.71-1.01)$ \\
\hline Bellomo et al. [7] & 21,090 & 20,921 & 14.3 & 10.6 & $0.74(0.70-0.79)$ \\
\hline Bellomo et al. [32] & 1,116 & 1,067 & 65.4 & 42.2 & $0.64(0.45-0.93)$ \\
\hline Jones et al. [30] & 16,246 & 104,001 & 53.7 & 39.1 & $0.73(0.68-0.78)$ \\
\hline Chan et al. [27] & 24,193 & 24,978 & 32.2 & 30.9 & $0.95(0.81-1.11)$ \\
\hline Konrad et al. [26] & 203,892 & 73,825 & 18.9 & 16.4 & $0.90(0.84-0.97)$ \\
\hline \multicolumn{6}{|l|}{ Salvatierra et al. [11] } \\
\hline Cluster-randomized study & 235,718 & 235,344 & 13.5 & 10.3 & $0.76(0.72-0.80)$ \\
\hline Priestley et al. [29] & 1,336 & 1,456 & 56.9 & 50.1 & $0.52(0.32-0.85)$ \\
\hline Hillman et al. [4] & 56,756 & 68,376 & 1.2 & 1.1 & $1.03(0.84-1.28)$ \\
\hline
\end{tabular}

RRT: rapid response team; OR: odds ratio; $\mathrm{Cl}$ : confidence interval. 


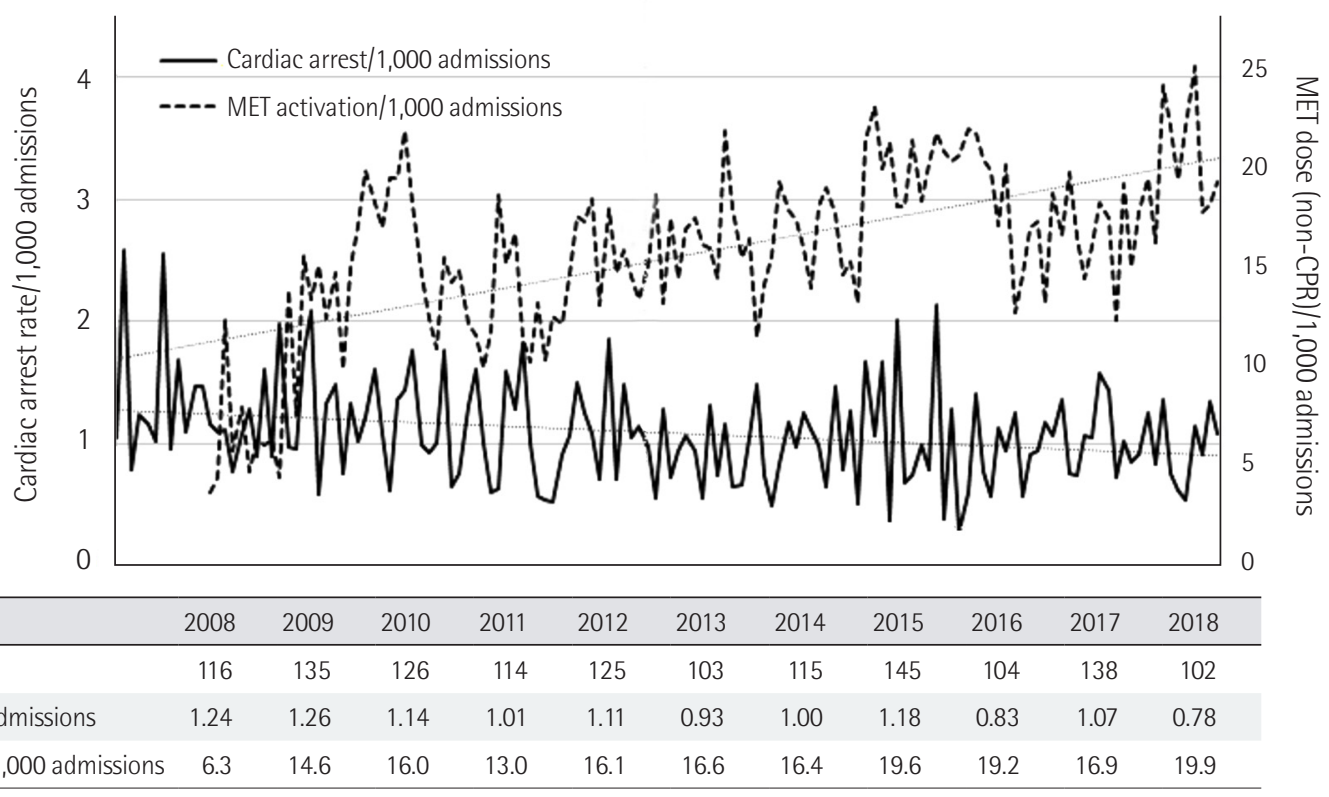

Figure 3. Relationship of rapid response system team activity and cardiac arrest rates in general ward. MET dose: number of rapid response team activation except CPR per 1,000 admissions; MET: medical emergency team; CPR: cardiopulmonary resuscitation. Unpublished data from Asan Medical Center.

tem by attending physician or nurse in the general ward, the MAT in Asan Medical Center is based on a surveillance program using an electronic medical recording (EMR) screening system [35]. Since MAT was implemented, several hospitals started to prepare for RRS. Samsung Medical Center also prepared an RRS and started pilot operation in January 2009, followed by Hanyang University Hospital in 2011 [36], Seoul National University Bundang Hospital in 2012 [37], and Seoul St. Mary's Hospital in 2013. Since then, awareness of RRS has been heightened due to promotions in several critical care-related conferences and academic meetings.

To implement RRS successfully, several barriers should be overcome, especially the culture. Korean culture is characterized by a somewhat shy and hierarchical relationship between doctors and nurses. Thus, the amount of direct calling for RRS is small at the beginning. Many Korean RRS overcame low calling rate by introducing EMR screening method including vital signs, labs, and proactive rounds (Figure 4).

Korean RRS was the topic of several papers during this period [35-42]. A single-center before-and after-study of RRS by Kwak et al. [36] and a Korean multicenter study [39] showed that RRS decreased in-hospital cardiac arrest. Kim et al. [38] compared in-hospital cardiac arrest rates by RRS working hours and showed that the incidence of in-hospital cardiac arrest was decreased only during RRS operating hours. RRS not only decreased in-hospital cardiac arrest rates, but also

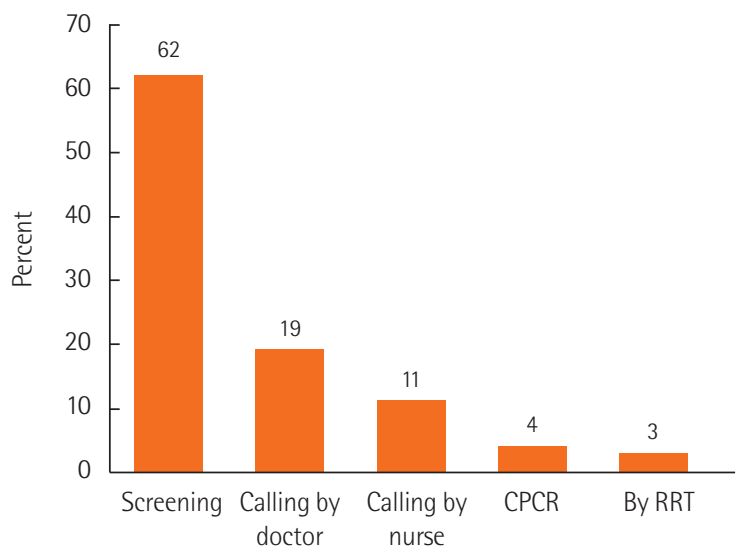

Figure 4. Types of activation of rapid response system from Korean multicenter data $(n=11,646)$. CPCR: cardiopulmonary cerebral resuscitation; RRT: rapid response team.

improved mortality from sepsis and first-attempt success rate of intubation (Figures 5, 6) [43,44]. However, many hospitals do not operate 24 hours a day, and physicians dedicated to such a team are rare. Most medical staff are working as intensivists and as members of the RRS team at the same time and are vulnerable to mental and physical exhaustion.

Leaders in hospitals know that an RRS is a good system. There is particular value in Korea where there are not enough medical personnel and resources. However, implementation of an RRS is potentially expensive, and there was no RRS manage- 


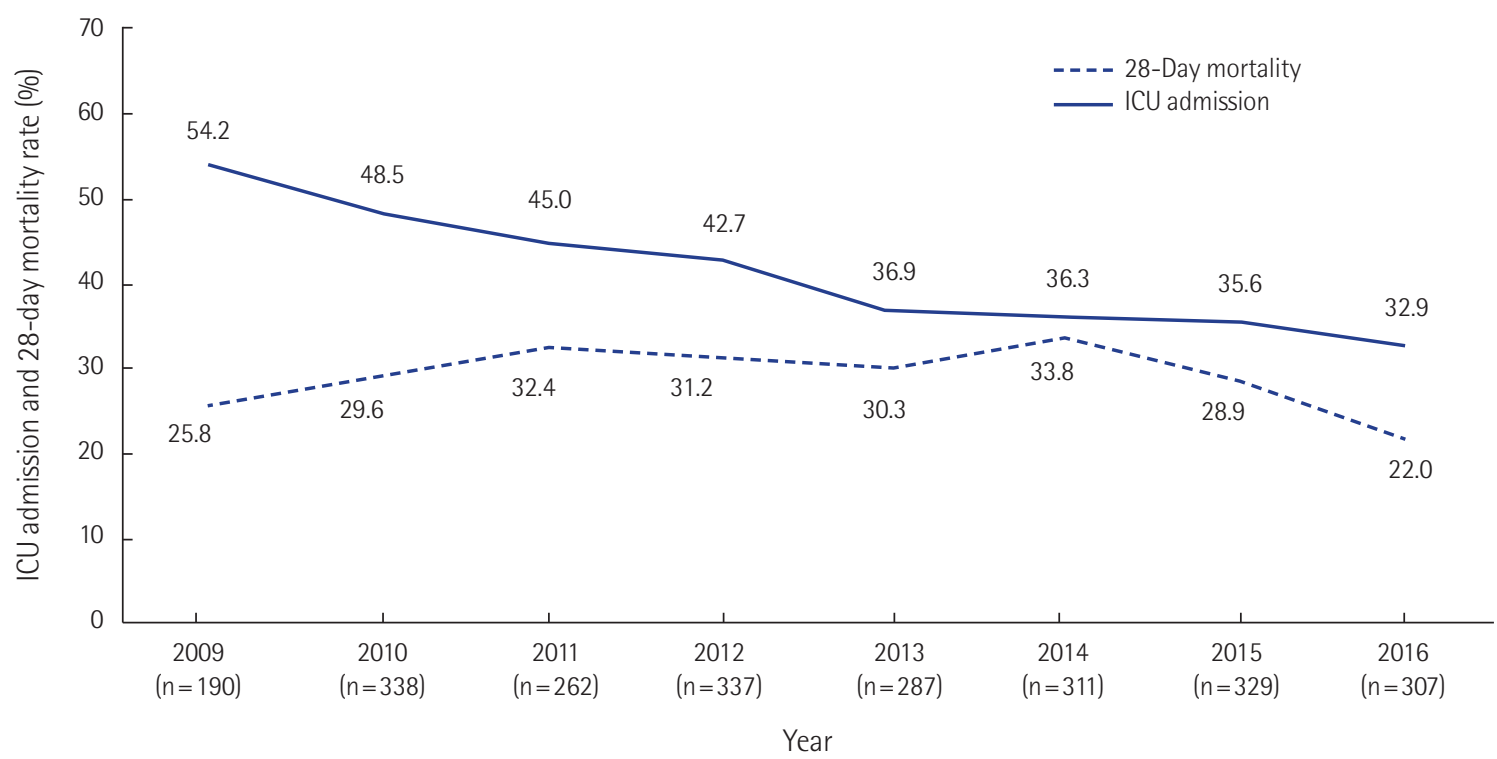

Figure 5. Outcomes of rapid response system activation in sepsis $(n=2,361)$. ICU: intensive care unit. Unpublished data from Asan Medical Center.

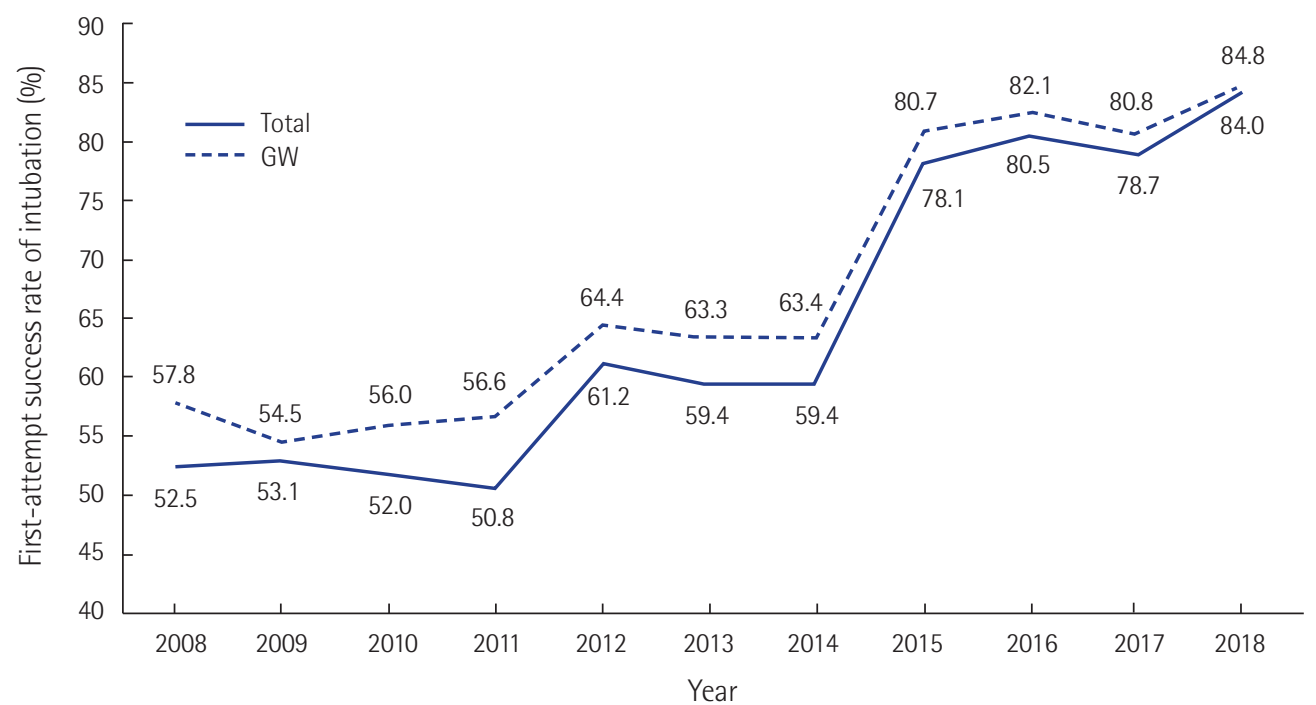

Figure 6. The first-attempt success rate of intubation has improved with the successful implementation of a rapid response system, in the general ward $(\mathrm{GW})$ and the entire hospital. A responding team in rapid response system included experienced operator for airway management. Unpublished data from Asan Medical Center.

ment fee until now. Thus, investment in RRS has been challenging. In 2018, as the Korea Institute for Healthcare Accreditation mandated items for patient safety improvement, more hospitals are adopting an RRS.

2019 is a milestone in the history of RRS in Korea. In May 2019, the Korean Health Insurance Review and Assessment Service and Ministry of Health and Welfare started an RRS pilot program, expanding RRSs further. The third edition revised accreditation standards for acute care hospital accreditation included implementation of an RRS. These changes are ex- pected to make RRS an essential part of improving patient safety. Through introduction of the RRS, hospital mortality rate, number of cardiac arrests, length of ICU stay, and rate of readmission to ICU may be reduced. This might lead to a reduction in overall medical costs by improving the quality of medical care.

\section{Future of RRS in Korea}

In 2019, more than 50 hospitals in Korea will run an RRS. Many hospitals are just beginning to overcome obstacles in imple- 
menting an RRS such as lack of awareness of the system, activation criteria, response protocol, organizational culture, resource scarcity, and fear of appearing inappropriate [45]. Constant, repeated education, feedback for quality improvement, and administrative support should be used to move forward. The optimal composition of RRS is not certain. It should be decided according to available institutional resources, preferences, and goals. Simulation training for RRS responders and hospital staffs can result in better management and communication. Appropriate calling and screening criteria as an afferent limb are essential. A novel informatics approach might be an option in the near future [46]. As wearable devices are more widely used, data from these may become available, leading to more accurate and timely assessment. As more hospitals adopt the RRS, associated Korean multicenter data will accumulate and will enable future development.

\section{CONCLUSIONS}

Since its introduction in the 2000s, the RRS has spread all over the world. Several studies have shown that this system is effective in reducing hospital mortality and in-hospital cardiac arrest. As it is becoming an essential part of the medical system for improving patient safety, clinicians need to understand the history and rationale of RRS and its potential benefits, limitations, and strategies for successful implementation. Although the history of RRS in Korea is not long, we have made great strides this year with administrative effort and support. With continued effort, we hope the RRS in Korea will evolve further in the future.

\section{CONFLICT OF INTEREST}

No potential conflict of interest relevant to this article was reported.

\section{ORCID}

Bo Young Lee https://orcid.org/0000-0001-7399-3822

Sang-Bum Hong https://orcid.org/0000-0003-2737-7695

\section{AUTHOR CONTRIBUTIONS}

Conceptualization, Data curation, \& Visualization: BYL, SBH. Writing-original draft: BYL. Writing-review \& editing: SBH.

\section{REFERENCES}

1. Neale G, Woloshynowych M, Vincent C. Exploring the causes of adverse events in NHS hospital practice. J R Soc Med 2001; 94:322-30

2. Jones DA, DeVita MA, Bellomo R. Rapid-response teams. N Engl J Med 2011;365:139-46.

3. Devita MA, Bellomo R, Hillman K, Kellum J, Rotondi A, Teres $D$, et al. Findings of the first consensus conference on medical emergency teams. Crit Care Med 2006;34:2463-78.

4. Hillman K, Chen J, Cretikos M, Bellomo R, Brown D, Doig G, et al. Introduction of the medical emergency team (MET) system: a cluster-randomised controlled trial. Lancet 2005;365: 2091-7.

5. Chan PS, Jain R, Nallmothu BK, Berg RA, Sasson C. Rapid response teams: a systematic review and meta-analysis. Arch Intern Med 2010;170:18-26.

6. Jones D, Bellomo R, DeVita MA. Effectiveness of the Medical Emergency Team: the importance of dose. Crit Care 2009;13: 313.

7. Bellomo R, Goldsmith D, Uchino S, Buckmaster J, Hart GK, Opdam H, et al. A prospective before-and-after trial of a medical emergency team. Med J Aust 2003;179:283-7.

8. Sebat F, Musthafa AA, Johnson D, Kramer AA, Shoffner D, Eliason $\mathrm{M}$, et al. Effect of a rapid response system for patients in shock on time to treatment and mortality during 5 years. Crit Care Med 2007;35:2568-75.

9. Buist M, Harrison J, Abaloz E, Van Dyke S. Six year audit of cardiac arrests and medical emergency team calls in an Australian outer metropolitan teaching hospital. BMJ 2007;335: 1210-2.

10. Jung B, Daurat A, De Jong A, Chanques G, Mahul M, Monnin $\mathrm{M}$, et al. Rapid response team and hospital mortality in hospitalized patients. Intensive Care Med 2016;42:494-504.

11. Salvatierra G, Bindler RC, Corbett C, Roll J, Daratha KB. Rapid response team implementation and in-hospital mortality. Crit Care Med 2014;42:2001-6.

12. Wilson RM, Runciman WB, Gibberd RW, Harrison BT, Newby L, Hamilton JD. The quality in Australian Health Care Study. Med J Aust 1995;163:458-71.

13. Schwendimann R, Blatter C, Dhaini S, Simon M, Ausserhofer D. The occurrence, types, consequences and preventability of in-hospital adverse events: a scoping review. BMC Health Serv Res 2018;18:521.

14. Schein RM, Hazday N, Pena M, Ruben BH, Sprung CL. Clinical antecedents to in-hospital cardiopulmonary arrest. Chest 1990;98:1388-92. 
15. Buist M, Bernard S, Nguyen TV, Moore G, Anderson J. Association between clinically abnormal observations and subsequent in-hospital mortality: a prospective study. Resuscitation 2004; 62:137-41.

16. Andersen LW, Kim WY, Chase M, Berg KM, Mortensen SJ, Moskowitz A, et al. The prevalence and significance of abnormal vital signs prior to in-hospital cardiac arrest. Resuscitation 2016;98:112-7.

17. Smith GB, Prytherch DR, Schmidt P, Featherstone PI, Knight D, Clements G, et al. Hospital-wide physiological surveillancea new approach to the early identification and management of the sick patient. Resuscitation 2006;71:19-28.

18. Levy MM, Evans LE, Rhodes A. The surviving sepsis campaign bundle: 2018 update. Crit Care Med 2018;46:997-1000.

19. Taenzer AH, Spence BC. The afferent limb of rapid response systems: continuous monitoring on general care units. Crit Care Clin 2018;34:189-98.

20. Subbe CP, Kruger M, Rutherford P, Gemmel L. Validation of a modified Early Warning Score in medical admissions. QJM 2001;94:521-6.

21. McGaughey J, Alderdice F, Fowler R, Kapila A, Mayhew A, Moutray M. Outreach and Early Warning Systems (EWS) for the prevention of intensive care admission and death of critically ill adult patients on general hospital wards. Cochrane Database Syst Rev 2007;(3):CD005529.

22. Royal College of Physicians. National Early Warning Score (NEWS) 2: standardising the assessment of acute-illness severity in the NHS. London: Royal College of Physicians; 2017.

23. Berwick DM, Calkins DR, McCannon CJ, Hackbarth AD. The 100,000 lives campaign: setting a goal and a deadline for improving health care quality. JAMA 2006;295:324-7.

24. The Joint Commission announces the 2009 National Patient Safety Goals and requirements. Jt Comm Perspect 2008;28:1, 11-5.

25. Kronick SL, Kurz MC, Lin S, Edelson DP, Berg RA, Billi JE, et al. Part 4: systems of care and continuous quality improvement. 2015 American Heart Association guidelines update for cardiopulmonary resuscitation and emergency cardiovascular care. Circulation 2015;132(18 Suppl 2):S397-413.

26. Konrad D, Jäderling G, Bell M, Granath F, Ekbom A, Martling CR. Reducing in-hospital cardiac arrests and hospital mortality by introducing a medical emergency team. Intensive Care Med 2010;36:100-6.

27. Chan PS, Khalid A, Longmore LS, Berg RA, Kosiborod M, Spertus JA. Hospital-wide code rates and mortality before and after implementation of a rapid response team. JAMA 2008;300: 2506-13.
28. Edelson DP, Yuen TC, Mancini ME, Davis DP, Hunt EA, Miller JA, et al. Hospital cardiac arrest resuscitation practice in the United States: a nationally representative survey. J Hosp Med 2014;9:353-7.

29. Priestley G, Watson W, Rashidian A, Mozley C, Russell D, Wilson J, et al. Introducing Critical Care Outreach: a ward-randomised trial of phased introduction in a general hospital. Intensive Care Med 2004;30:1398-404.

30. Jones D, Bellomo R, Bates S, Warrillow S, Goldsmith D, Hart $\mathrm{G}$, et al. Long term effect of a medical emergency team on cardiac arrests in a teaching hospital. Crit Care 2005;9:R808-15.

31. Buist MD, Moore GE, Bernard SA, Waxman BP, Anderson JN, Nguyen TV. Effects of a medical emergency team on reduction of incidence of and mortality from unexpected cardiac arrests in hospital: preliminary study. BMJ 2002;324:387-90.

32. Bellomo R, Goldsmith D, Uchino S, Buckmaster J, Hart G, Opdam $\mathrm{H}$, et al. Prospective controlled trial of effect of medical emergency team on postoperative morbidity and mortality rates. Crit Care Med 2004;32:916-21.

33. Maharaj R, Raffaele I, Wendon J. Rapid response systems: a systematic review and meta-analysis. Crit Care 2015;19:254.

34. ANZICS-CORE MET dose investigators. Mortality of rapid response team patients in Australia: a multicentre study. Crit Care Resusc 2013;15:273-8.

35. Huh JW, Lim CM, Koh Y, Lee J, Jung YK, Seo HS, et al. Activation of a medical emergency team using an electronic medical recording-based screening system. Crit Care Med 2014;42: 801-8.

36. Kwak HJ, Yun I, Kim SH, Sohn JW, Shin DH, Yoon HJ, et al. The extended rapid response system: 1-year experience in a university hospital. J Korean Med Sci 2014;29:423-30.

37. Lee YJ, Park JJ, Yoon YE, Kim JW, Park JS, Kim T, et al. Successful implementation of a rapid response system in the department of internal medicine. Korean J Crit Care Med 2014;29: 77-82.

38. Kim Y, Lee DS, Min H, Choi YY, Lee EY, Song I, et al. Effectiveness analysis of a part-time rapid response system during operation versus nonoperation. Crit Care Med 2017;45:e592-9.

39. Park Y, Ahn JJ, Kang BJ, Lee YS, Ha SO, Min JS, et al. Rapid response systems reduce in-hospital cardiopulmonary arrest: a pilot study and motivation for a nationwide survey. Korean J Crit Care Med 2017;32:231-9.

40. Song JU, Suh GY, Park HY, Lim SY, Han SG, Kang YR, et al. Early intervention on the outcomes in critically ill cancer patients admitted to intensive care units. Intensive Care Med 2012;38: 1505-13.

41. Lee SH, Leem AY, Nho Y, Kim YA, Kim KD, Kim YS, et al. A pi- 
lot study of the effectiveness of medical emergency system implementation at a single center in Korea. Korean J Crit Care Med 2017;32:133-41.

42. Kim SW, Lee HY, Han MR, Lee YS, Kang EH, Jang EJ, et al. Epidemiology and clinical characteristics of rapid response team activations. Korean J Crit Care Med 2017;32:124-32.

43. Lee DH, Han M, An JY, Jung JY, Koh Y, Lim CM, et al. Video laryngoscopy versus direct laryngoscopy for tracheal intubation during in-hospital cardiopulmonary resuscitation. Resuscitation 2015;89:195-9.
44. Baek MS, Han M, Huh JW, Lim CM, Koh Y, Hong SB. Video laryngoscopy versus direct laryngoscopy for first-attempt tracheal intubation in the general ward. Ann Intensive Care 2018; 8:83.

45. Lyons PG, Edelson DP, Churpek MM. Rapid response systems. Resuscitation 2018;128:191-7.

46. Lee Y, Kwon JM, Lee Y, Park H, Cho H, Park J. Deep learning in the medical domain: predicting cardiac arrest using deep learning. Acute Crit Care 2018;33:117-20. 